\title{
Evaluation of a Therapeutic Alternative for Telogen Effluvium: A Pilot Study
}

\author{
Alfredo Rossi ${ }^{1}$, Lara Priolo ${ }^{1}$, Alessandra Iorio ${ }^{1}$, Enrica Vescarelli ${ }^{2}$, Martina Gerardi ${ }^{1}$, Daniele Campo ${ }^{3}$, \\ Donato Di Nunno $^{1}$, Simona Ceccarelli ${ }^{2}$, Stefano Calvieri ${ }^{1}$, Antonio Angeloni ${ }^{4}$, Cinzia Marchese ${ }^{2}$ \\ ${ }^{1}$ Department of Internal Medicine and Medical Specialties, Sapienza University of Rome, Rome, Italy; ${ }^{2}$ Department of Experimental \\ Medicine, Sapienza University of Rome, Rome, Italy; ${ }^{3}$ Italian Orthodermic Institute, Rome, Italy; ${ }^{4}$ Department of Molecular Medi- \\ cine, Sapienza University of Rome, Rome, Italy. \\ Email: cinzia.marchese@uniroma1.it
}

Received May $29^{\text {th }}, 2013$; revised July $1^{\text {st }}, 2013$; accepted July $10^{\text {th }}, 2013$

Copyright (C) 2013 Alfredo Rossi et al. This is an open access article distributed under the Creative Commons Attribution License, which permits unrestricted use, distribution, and reproduction in any medium, provided the original work is properly cited.

\begin{abstract}
Background/Aim: Telogen effluvium (TE) is a scalp disorder characterized by the thinning or shedding of hair resulting from the early entry of hair in the telogen phase. Nigella sativa (NS) is a dicotyledonous belonging to the Ranunculacae family. It has been shown that its major constituent, tymoquinon (TQ), exerts anti-oxidant and anti-inflammatory effects by inhibiting pro-inflammatory mediators, such as cyclooxygenase and prostaglandin D2. The aim of this study is to evaluate the efficacy of NS essential oil as a potential treatment for TE, a pathology characterized by a significant inflammatory component. Study Design/Methods: Twenty patients affected by TE for this double-blind, placebo controlled and randomized study were enrolled. Ten of these patients were treated with a scalp lotion containing $0.5 \%$ NS, daily for 3 months, while the other ten patients were treated with placebo daily for 3 months. Videodermatoscopic analysis (Trichoscan Dermoscope Fotofinder ${ }^{\circledR}$ ) and evaluation of three independent dermatologists were performed before treatment (T0), after 3 months of treatment (T3) and at the 6 months follow-up (T6). Results: The results showed a significant improvement in $70 \%$ of patients treated with NS. Videodermatoscopic analysis showed a significant increment of hair density and hair thickness in patients treated with NS. NS was also able to reduce the inflammation observed in the majority of patients affected by TE. Conclusions: The results of this study suggest that NS can be considered potentially useful for the treatment of TE.
\end{abstract}

Keywords: Telogen Effluvium; Nigella sativa; Hair Density; Hair Thickness

\section{Introduction}

The term telogen effluvium (TE), first coined by Kligman in 1961 [1], refers to the loss of club (telogen) hair in disease states of the follicle. TE is characterized by abnormality of hair cycling with excessive loss of telogen hairs and it shows a heterogeneous etiology. To establish the cause of the hair loss, one requires a history to identify known triggers, biochemical investigations to exclude endocrine, nutritional or autoimmune etiologies and in many cases histology to identify the earliest stages of androgenetic alopecia. Pathogenic mechanisms of TE were suggested by Headington, who described five functional types of TE based on different phases of the follicular cycle: anagen release, delayed anagen release, short anagen syndrome, immediate telogen release, and delayed telogen release [2]. Early teloptosis, conveying teloptosis and early initiation of telogen represent the main mechanisms involved in hair loss in telogen [3].

Depending on the length of time, this increased shedding persists, it is referred to as an acute or chronic TE. In acute TE, the shedding can persist for up to 6 months. Acute TE usually occurs $2-3$ months after a triggering event (major surgery, injury, severe illness, childbirth, crash diet and numerous medications) [4]. In about 33\% of cases of acute TE, no triggering events can be identified [2]. The pull test is positive ( $>6$ hairs in telogen) and the trichogram usually shows more than $25 \%$ of telogen hairs [1]. Histological analysis of acute TE shows a normal number of hair follicles with no miniaturization, and resembles normal scalp [5]. The acute form is a self-limiting condition lasting for about 3-6 months, but if the triggering event persists, the acute form can evolve in chronic condition. When the shedding lasts more than 6 months, or persistently cycles in and out of periods of 
increased shedding, it is called a chronic TE. A chronic TE can last for years and it is more often reported in women than men [6]. The accepted causes of this chronic condition are thyroid disorder, profound iron-deficiency anemia, malnutrition, drugs and chronic autoimmune diseases. Hair shedding involves the entire scalp. Moreover, there is a moderate bitemporal recession, although it is possible to observe short and thinned hairs recently formed in the temporal region. One of the related symptoms is trichodynia. Also in chronic TE, hair follicles do not present number variations, but there is an increased number of telogen hairs $(20 \%-30 \%$, with respect to $5 \%$ $-10 \%$ of the normal scalp), and some evidence of miniaturization if it is superimposed on an evolving androgenic alopecia [5]. The diagnosis of TE requires scalp examination, hair pull test, full blood count, thyroid function test, syphilis serology and investigation of nutritional status (serum zinc, serum iron) [3]. Although the etiopathogenesis of TE is largely unknown, chronic stress may represent one of the major causes, since it is known that it exerts a profound inhibitory effect on hair growth $[7,8]$. In particular, the corticotropin-releasing factor (CRF), which is involved in stress response, is able to interrupt hair follicle growth cycle in humans and mice [9-11]. Occasional anagen dystrophy, trichodynia [12] and response to corticosteroids suggest an inflammatory nature for TE, which is also indicated by the presence of substance P (SP). SP, a member of the tachykinin neuropeptide family, is one of the strongest mediators of neurogenic inflammation $[13,14]$, which acts through the activation of neurokinin 1 receptor (NK1R) and is involved in several inflammatory conditions $[15,16]$. SP was identified as a key mediator of stress-induced hair growth inhibition [17]. In fact, it activates the secretion of inflammatory cytokines, such as tumor necrosis factor- $\alpha$ (TNF- $\alpha$ ), interleukin- $1 \beta$ (IL- $1 \beta$ ) and interferon- $\gamma$ (IFN- $\gamma$ ), from macrophages and mast cells. This inflammatory context may play a role in the damage of hair follicle morphology through up-regulation of apoptosis [18].

Nigella sativa (NS), commonly known as black cumin, is an annual flowering plant native to Mediterranean countries, Pakistan and India [19]. Thymoquinone (TQ), dithymoquinone (DTQ), thymohydroquinone (THQ), and thymol (THY) are the principal pharmacologically active constituents of NS. NS seeds and its oil extract have been demonstrated to exert anti-tumoral, anti-oxidant and antiinflammatory activities in a number of diseases [20-22]. $\mathrm{TQ}$, which represents the predominant component $(30 \%$ $48 \%$ ) of NS, has been reported to inhibit inflammatory cell infiltration in the brain through inhibition of NF-kB activation [23]. TQ shows an anti-inflammatory activity through the inhibition of cyclooxygenase 2 (COX-2) expression and prostaglandin D2 (PGD2) synthesis, which would explain why NS has been considered a natural remedy for many diseases and inflammatory conditions. Moreover, recent studies revealed an important inhibitory role of PGD2 on hair follicle [24]: in fact, its binding to the GPR44 receptor expressed in the hair follicle seemed to induce miniaturization of the follicle, thus contributing to the development and maintenance of vellus-like hair and probably preventing the maturation of stem cells into progenitor cells and of vellus-like hair into normal, thick and pigmented, terminal hair. Therefore, NS inhibition of activation and synthesis of NF-kB and of PDG2 can contribute to the regulation of hair cycle [25].

Considering this, we suggested the use of NS as a possible treatment for inflammation pathologies, such as TE.

\section{Materials and Methods}

\subsection{Patients}

Twenty women affected by acute TE, aged between 22 and 50 years (mean age $36 \pm 8$ ) were enrolled in this study. Principal exclusion criteria included: use of minoxidil within 1 year; use of drugs with androgenic or anti-androgenic properties; use of drugs that can lead hair loss; alopecia caused by surgery on the scalp; refusal to provide informed consent or to comply with the study protocol.

All patients were fully informed about the purpose and duration of this study and they were free to leave the study at any time. The treatment with NS was planned to be withdrawn in case of observation of adverse event. Written informed consent was obtained from all the participants. The study protocol conformed to the guidelines of the 1975 Declaration of Helsinki, as revised in 1983, and was approved by the research ethics committee of Sapienza University of Roma.

\subsection{Study Design}

Ten out of the twenty patients were provided with a topic formulation containing $0.5 \%$ NS essential oil, $3 \%$ glycerin, $0.4 \%$ lavender essential oil and $60 \%$ alcohol to 100 $\mathrm{ml}$. The other ten patients were provided with a topic formulation containing the same ingredients except for NS essential oil (placebo). Each patient was randomized by a computer-generated list to receive topic formulations $(2 \mathrm{ml} /$ day) containing either NS or placebo. Both the patient and dermatologist were "blinded" to the group assignment. Marked formulation packs (180 ml solution for 3 months supply) were designated for each patient. Patients accepted to comply with the study protocol by repeating the treatment daily, with $2 \mathrm{ml}$ of formulation each day, for 3 months. Follow-up visits were scheduled after 3 and 6 months. High resolution digital photographs and photo-trichograms at baseline (T0), after 3 months of treatment (T3) and at the 6 months follow-up (T6) were 
taken.

\subsection{Clinical Outcomes Evaluation}

The efficacy of treatment was assessed through evaluation of changes from baseline in hair density and thickness and through global photographic assessment by dermatologists. Such analyses were completed before the randomization code was broken at the end of the study.

Determination of hair density was performed on microscopic images acquired in an area of approximately $1 \mathrm{~cm}^{2}$ in the vertex region with Trichoscan Dermoscope Fotofinder ${ }^{\circledR}$ (Teachscreen Software, Bad Birnbach, Germany) using lenses with 20 or 70 factor of magnification and saved in the database. The same procedure was repeated before the treatment (T0), after 3 months of treatment (T3) and after 6 months (T6). Trichoscan analysis software automatically calculated the number of hairs and hair density per square centimeter. The mean thickness of the hairs was also measured at baseline (T0), after 3 month of treatment (T3) and at the 6 months follow-up (T6). Dermoscopic examination of hair loss areas on the scalp of patients was also performed to analyze the presence of inflammatory pattern, such as severe itching, redness of the scalp and peripilar signs, corresponding to perifollicular pigmentations.

The evaluation of treatment was made by assessment of standardized global photographs. The hairs of patients were centered-parted to take each photographic view. All the photos were registered before entering the study (T0), after 3 months of treatment (T3) and at the 6 months follow-up (T6). The degree of improvement was graded by three independent dermatologists who did not participate in the medical care of the patients and who were blinded to the treatment. The effectiveness of the treatment was evaluated by assigning a score, for each patient, compar- ing photographs separately, based on a 7-point scale: -3 $=$ greatly decreased, $-2=$ moderately decreased, $-1=$ slightly decreased, $0=$ no change, $1=$ slightly increased, 2 = moderately increased, $3=$ greatly increased. We considered the average value among the scores assigned by each dermatologist.

\section{Results}

All the enrolled patients completed 3 months treatment and 6 months follow-up.

\subsection{Evaluation of Hair Density}

The aim of this study was to examine the difference between patients treated with NS and placebo controls with regard to changes in hair density after 3 months of treatment (T3) and at the 6 months follow-up (T6). At T3, $90 \%$ of patients using NS solution demonstrated a significant increase in the number of hairs $/ \mathrm{cm}^{2}$ in comparison to baseline (T0), while in the group of patients treated with placebo, only $30 \%$ of patients showed hair density increase (Table 1, Figures 1(a), (b)). In detail, nine out of ten patients belonging to the NS group showed an increase in hair number. Five of these patients reported a further increase at T6, while in four patients we observed a stabilization of the disease with a preservation of hair density also at follow-up. Results strongly differed from the placebo group, where six out of ten patients displayed severe hair loss at both $\mathrm{T} 3$ and $\mathrm{T} 6$, only three patients showed an improvement in hair density, and one patient showed a stabilization of the disease with the same hair density at T0 and T3 (Table 1).

\subsection{Evaluation of Cumulative Hair Thickness}

We assessed cumulative hair thickness, an additional

Table 1. Hair density $/ \mathrm{cm}^{2}$ in patients treated with NS or placebo at the baseline (T0), after 3 months of treatment (T3) and at the 6 months follow-up (T6).

\begin{tabular}{|c|c|c|c|c|c|c|c|}
\hline \multirow{2}{*}{ Patient N. } & \multicolumn{3}{|c|}{ NS group } & \multirow{2}{*}{ Patient N. } & \multicolumn{3}{|c|}{ Placebo group } \\
\hline & T0 & $\mathrm{T} 3$ & T6 & & T0 & T3 & T6 \\
\hline 2 & 179 & 182 & 182 & 1 & 146 & 144.5 & 142 \\
\hline 3 & 232.5 & 242.5 & 263 & 5 & 105 & 102 & 102 \\
\hline 4 & 106 & 127.5 & 127.5 & 6 & 132.5 & 127.5 & 117.5 \\
\hline 8 & 202 & 205 & 205 & 7 & 220 & 174 & 143.5 \\
\hline 10 & 182 & 197.5 & 198.5 & 9 & 213.5 & 215 & 217.5 \\
\hline 11 & 221 & 223 & 225.5 & 13 & 182 & 176.5 & 176.5 \\
\hline 12 & 228.5 & 229 & 229 & 14 & 198.5 & 190 & 180.5 \\
\hline 15 & 251.5 & 247.5 & 247.5 & 16 & 176 & 178.5 & 182 \\
\hline 19 & 133.5 & 137 & 145 & 17 & 172 & 178.5 & 182 \\
\hline 20 & 224 & 245.5 & 246 & 18 & 115.5 & 115.5 & 119 \\
\hline
\end{tabular}



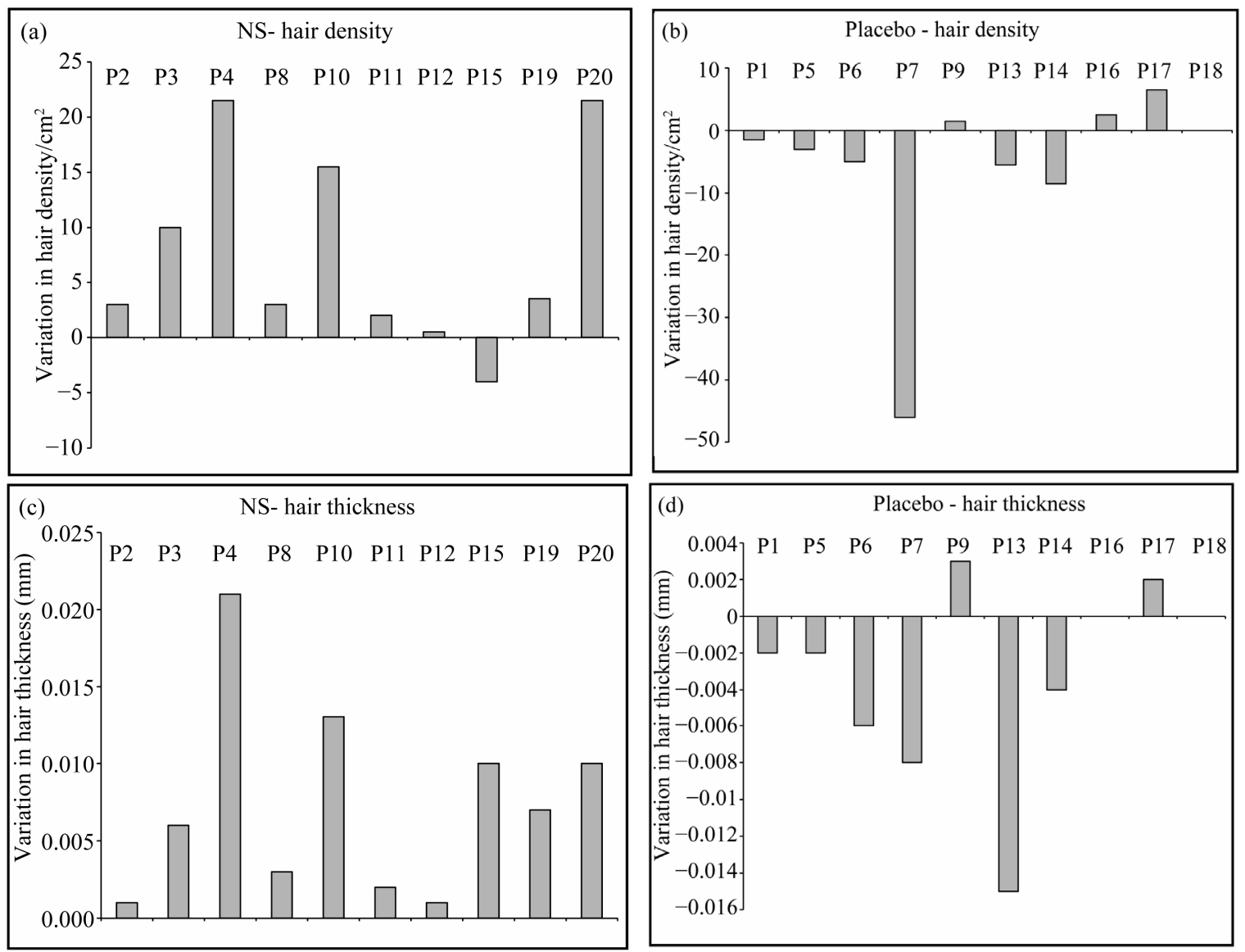

Figure 1. (a), (b) Graphical representation of the variation in hair density/cm in patients treated with NS (a) or placebo (b) after 3 months of treatment; (c), (d) Graphical representation of the variation in hair thickness (mm) in patients treated with NS (c) or placebo (d) after 3 months of treatment.

useful parameter to estimate the real efficacy of the treatment. After 3 months of treatment, all patients in NS group showed a relevant increase in cumulative hair thickness. In fact, also in the three patients who showed no relevant variation in hair density, we observed a positive variation in cumulative hair thickness. Moreover, the evaluation after 6 months showed a preservation or further improvement of hair caliber in all patients (Table 2, Figure 1(c)). Conversely, in the placebo group cumulative hair thickness was decreased in $60 \%$ of patients, while only $20 \%$ showed an increase in cumulative hair thickness and $20 \%$ showed no variations in hair caliber (Table 2, Figure 1(d)).

\subsection{Determination of NS Effects on Scalp Inflammation}

We also evaluated the possible presence of an inflammatory pattern, represented by severe itching, redness of the scalp or perifollicular lymphocytic infiltrates around the bulb hair (peripilar signs). Symptoms of inflammation were recognizable in almost all patients, and in particular we identified the presence of peripilar sign in $60 \%$ of our patients. Our results confirmed that patients subjected to NS treatment underwent a significant improvement of the inflammatory state, with a complete regression of peripilar sign (Figure 2(a)). Disappearance of perifollicular inflammation in NS-treated patients was also accompanied by reduction of one of the main inflammatory symptoms, trichodynia. Conversely, patients treated with placebo still maintained an inflammatory condition at the 6 months follow-up (Figure 2(b)).

\subsection{Determination of NS Effects by Global Photographic Assessment}

Clinical efficacy of NS was checked after 3 months of daily topical application of the mixture to the scalp (T3) and at the 6 months follow-up (T6). Patients treated with NS or placebo were evaluated by three independent dermatologists who classified all patients using a 7-point scale, by analyzing pictures taken 3 months after treatment (T3) and at the 6 months follow-up (T6), in comparison with pictures taken at the baseline before treatment (T0).

In the NS group, dermatologists graded three patients 
Table 2. Cumulative hair thickness (mm) in patients treated with NS or placebo at the baseline (T0), after 3 months of treatment (T3) and at the 6 months follow-up (T6).

\begin{tabular}{|c|c|c|c|c|c|c|c|}
\hline \multirow{2}{*}{ Patient N. } & \multicolumn{3}{|c|}{ NS group } & \multirow{2}{*}{ Patient N. } & \multicolumn{3}{|c|}{ Placebo group } \\
\hline & $\mathrm{T} 0$ & $\mathrm{~T} 3$ & T6 & & T0 & $\mathrm{T} 3$ & T6 \\
\hline 2 & 0.062 & 0.063 & 0.064 & 1 & 0.070 & 0.068 & 0.087 \\
\hline 3 & 0.081 & 0.087 & 0.087 & 5 & 0.067 & 0.065 & 0.065 \\
\hline 4 & 0.077 & 0.098 & 0.098 & 6 & 0.059 & 0.053 & 0.049 \\
\hline 8 & 0.078 & 0.081 & 0.082 & 7 & 0.062 & 0.054 & 0.034 \\
\hline 10 & 0.075 & 0.088 & 0.088 & 9 & 0.080 & 0.083 & 0.094 \\
\hline 11 & 0.075 & 0.077 & 0.077 & 13 & 0.075 & 0.060 & 0.060 \\
\hline 12 & 0.076 & 0.077 & 0.077 & 14 & 0.067 & 0.063 & 0.053 \\
\hline 15 & 0.052 & 0.062 & 0.063 & 16 & 0.071 & 0.071 & 0.069 \\
\hline 19 & 0.078 & 0.085 & 0.085 & 17 & 0.079 & 0.081 & 0.085 \\
\hline
\end{tabular}
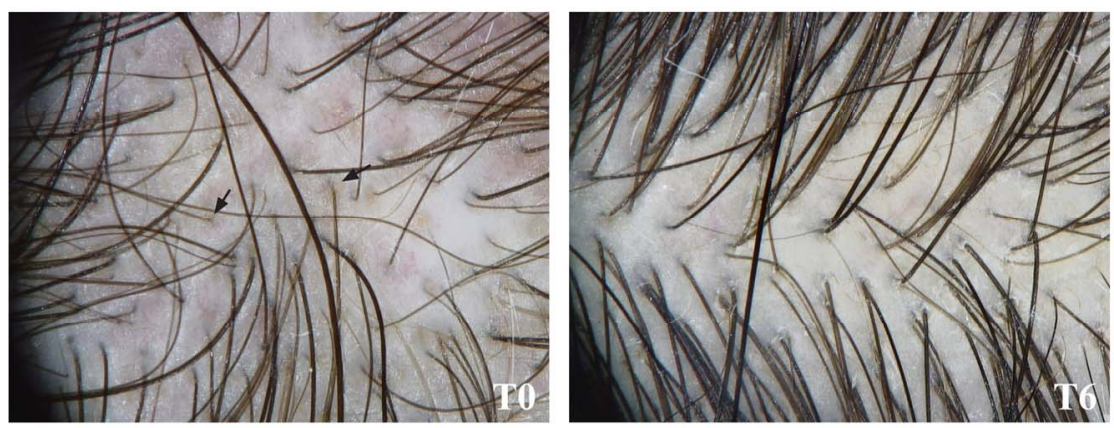

(a)
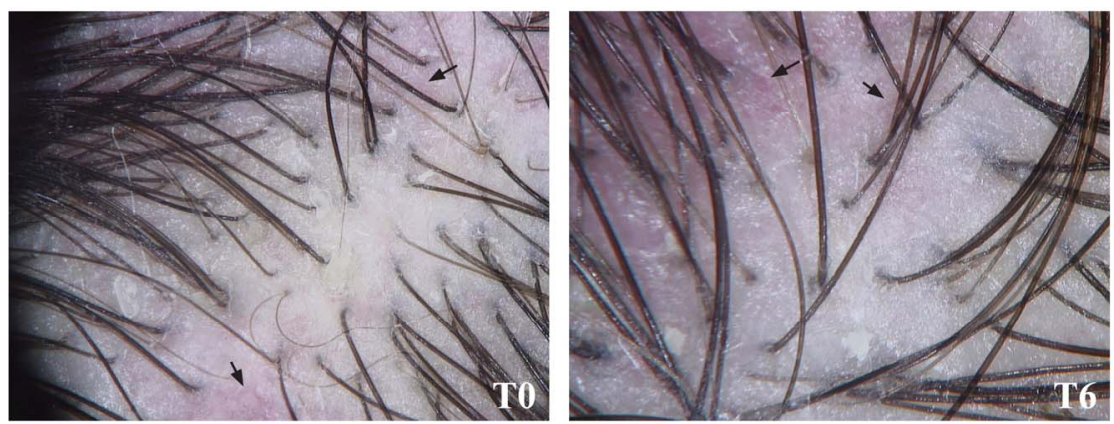

(b)

Figure 2. Inflammatory signs in patients affected by TE. (a) Patients treated with NS showed complete regression of peripilar signs at the 6 months follow-up (T6), compared to baseline (T0); (b) Patients treated with placebo showed persistent scalp inflammation at the 6 months follow-up (T6), compared to baseline (T0).

as steady, three patients as slightly improved, two patients as moderately improved and two patients as highly improved. These conditions were not changed at the 6 months follow-up (Table 3, Figure 3(a)). Patients treated for 3 months with placebo (T3) were classified as follow: two patients as slightly improved, two patients as stable, four patients as slightly worsened and two patients as moderately worsened. At the 6 months follow-up, two patients maintained the same condition than in T3, three patients reported a slight improvement with respect to T3, thus indicating the beginning of a spontaneous resolution of acute TE, and five patients presented a worsened condition, predictive of TE chronicization (Table 3, Figure 3(b)).

\section{Discussion}

Telogen effluvium is a hair cycling alteration with an excessive loss of telogen hairs. In acute TE, patients presented a sudden and massive hair loss, generally 2 - 3 
Table 3. Summary of patients' outcomes assessed 3 months after treatment (T3) and at the 6 months follow-up (T6). For each patient is reported the mean of the scores assigned by three independent dermatologists using a 7-point checklist.

\begin{tabular}{cccccc}
\hline \multirow{2}{*}{ Patient N. } & \multicolumn{2}{c}{ NS group } & & \multicolumn{2}{c}{ Placebo group } \\
\cline { 2 - 3 } & T3 & T6 & & T3 & T6 \\
\hline 2 & 0 & 0 & 1 & -1 & -2 \\
3 & +1 & +1 & 5 & -1 & -1 \\
4 & +3 & +3 & 6 & -1 & -2 \\
8 & +1 & +1 & 7 & -2 & -3 \\
10 & +2 & +2 & 9 & +1 & +2 \\
11 & 0 & 0 & 13 & -2 & -2 \\
12 & 0 & 0 & 14 & -1 & -2 \\
15 & +1 & +1 & 16 & 0 & -1 \\
19 & +3 & +3 & 17 & +1 & +2 \\
20 & +2 & +2 & 18 & 0 & +1 \\
\hline
\end{tabular}
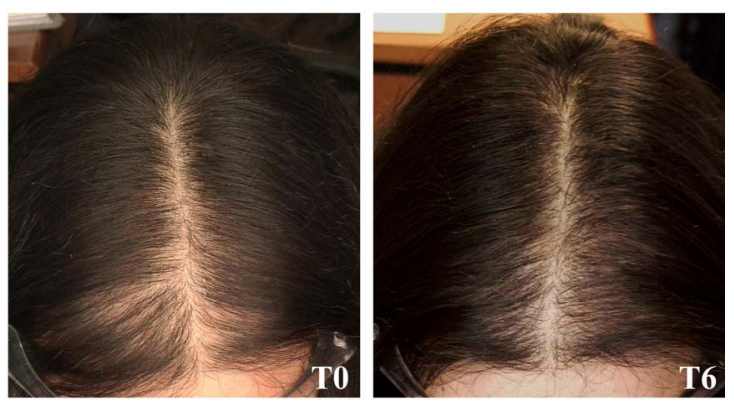

(a)
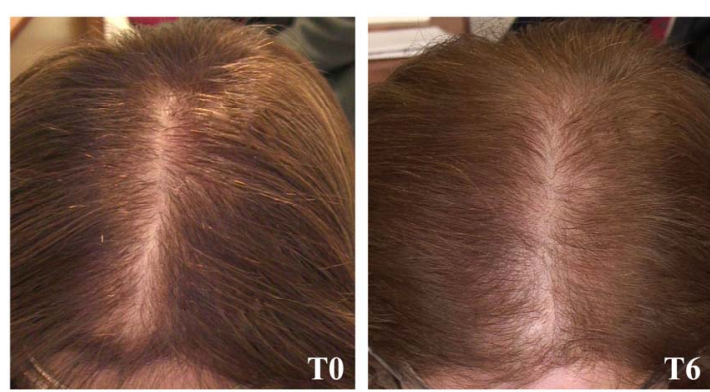

(b)

Figure 3. Clinical photographs of representative patients treated with NS (a) or placebo (b), at baseline (T0) and at the 6 months follow-up (T6).

months subsequent to a triggering event. If these triggering factors are prolonged, shedding hairs can develop insidiously and lead to chronic TE. Minoxidil, a potent antihypertensive agent, represents the most frequent topical treatment [26]. Nevertheless, this drug causes some adverse effects, such as scalp irritation, including dryness, scaling, itching, and/or redness [27]. Most cases $(82 \%)$ of scalp irritation after treatment with minoxidil resulted from a contact dermatitis to propylene glycol, a vehicle of the solution [28]. Moreover, such treatment needs to be prolonged indefinitely to maintain a response. Furthermore, since this drug acts by altering hair cycling, causing premature termination of telogen phase and probably prolonging anagen [29], minoxidil treatment could be disadvantageous in TE, causing an increase in hair loss due to the synchronization of the hair cycle.

NS is a plant with a wide spectrum of pharmacological actions, such as anti-diabetic, immunomodulatory, analgesic, anti-inflammatory and antioxidant effect [22]. It has been demonstrated that administration of NS seed extract intraperitoneally to rats does not affect hepatic and renal function [30]. No mortality or over-toxicity has been observed during oral administration of the seed oil in rats and mice, even at high doses [31]. Thymoquinone, the most representative component of NS, showed antiinflammatory effects since it is able to suppress proinflammatory cytokines such as IL- $1 \beta$, TNF- $\alpha$ [32], IL-4, IL-5, IL-3 [33]. Several studies tested this substance in different conditions, such as cancer [22] and inflammatory diseases [34].

Considering that TE is characterized by a high inflammatory component and that NS possess well-known anti-inflammatory properties, we decided to evaluate the effects of NS in the treatment of TE. The aim of this double blind, placebo controlled, randomized study was to obtain a reduction of inflammatory compounds in order to re-establish hair cycle and to reduce the healing time in patients affected by acute TE. Our data demonstrated NS efficacy by an improvement of hair density and hair thickness after 3 months of treatment (T3). In particular, $70 \%$ of patients showed an improvement, with increase in hair growth, while $30 \%$ of patients showed no change and none showed TE worsening. In the placebo group, $50 \%$ of patients showed worsening of clinical condition, $20 \%$ showed no change and developed chronic TE after 6 months and only $20 \%$ showed a partial improvement.

Moreover, we also documented the presence of a scalp inflammation in patients affected by TE, with evidence of perifollicular inflammatory infiltrate in $60 \%$ of our patients, indicated by the finding of the so-called peripilar sign, first described by Deloche et al. [35] as a brown or brown-gray circular, perifollicular discoloration, linked to superficial perifollicular lymphocytic infiltrates. Although the mechanism leading to the pigmentation of the perifollicular skin surface is still unknown, the peripilar sign may be observed in persons with androgenetic alopecia and, occasionally, in TE [35]. We were able to demonstrate that NS treatment provide an improvement of scalp inflammation, with disappearance of peripilar sign and other symptoms related to inflammatory pattern.

Previous studies reported few cases of allergic contact 
dermatitis following topical application of NS oil [36, 37], but a more recent study pointed out the absence of cutaneous adverse effect with $2 \%$ NS topical treatment [38]. In our study, for all the duration of treatment, no patients developed cutaneous adverse effect or allergy to the substance.

In our study, the lack of efficacy found in some patients $(30 \%$ of patients who did not take advantage from NS treatment) might be partially related to failure of compliance in these patients. Moreover, since TE shows a heterogeneous etiology, it is possible that in some of these patients the pathology did not present a strong inflammatory component, thus limiting the effectiveness of NS treatment.

\section{Conclusion}

In conclusion, our results confirmed data of previous studies about anti-inflammatory properties of NS and its ability in reducing symptoms, such as trichodynia, typical of patients affected by TE. Moreover, the results obtained indicated that NS is effective in promoting hair growth, thus inducing a significant improvement in terms of hair density and caliber. Considering this, we suggest that topical use of NS for TE treatment can be considered as a valid alternative to traditional therapies such as minoxidil.

\section{REFERENCES}

[1] A. M. Kligman, "Pathologic Dynamics of Human Hair Loss: Telogen Effluvium," Archives of Dermatology, Vol. 83, 1961, pp. 175-198. doi:10.1001/archderm.1961.01580080005001

[2] J. T. Headington, "Telogen Effluvium," Archives of Dermatology, Vol. 129, No. 3, 1993, pp. 356-363. doi:10.1001/archderm.1993.01680240096017

[3] A. Rebora, "Telogen Effluvium: An Etiopathogenetic Theory," International Journal of Dermatology, Vol. 32, No. 5, 1993, pp. 339-340. doi:10.1111/j.1365-4362.1993.tb01468.x

[4] D. Whiting, S. F. Templeton and A. R. Solomon, "Disorders of Cutaneous Appendages," In: R. L. Barnhill, Ed., Textbook of Dermatopathology, McGraw-Hill, New York, 1998, pp. 201-223.

[5] G. Eudy and A. R. Solomon, "The Histopathology of Noncicatricial Alopecia," Seminars in Cutaneous Medicine and Surgery, Vol. 25, No. 1, 2006, pp. 35-40. doi:10.1016/j.sder.2006.01.005

[6] D. A. Whiting, "Chronic Telogen Effluvium: Increased Scalp Hair Shedding in Middle Aged Women," Journal of the American Academy of Dermatology, Vol. 35, No. 6, 1996, pp. 899-906. doi:10.1016/S0190-9622(96)90113-9

[7] P. C. Arck, A. Slominski, T. C. Theoharides, E. M. Peters and R. Paus, "Neuroimmunology of Stress: Skin Takes Center Stage," Journal of Investigative Dermatology, Vol. 126, No. 8, 2006, pp. 1697-1704.

\section{doi:10.1038/sj.jid.5700104}

[8] N. Ito, T. Ito, A. Kromminga, A. Bettermann, M. Takigawa, F. Kees, R. H. Straub and R. Paus, "Human Hair Follicles Display a Functional Equivalent of the Hypothalamic-Pituitaryadrenal Axis and Synthesize Cortisol," FASEB Journal, Vol. 19, No. 10, 2005, pp. 1332-1334.

[9] E. M. Peters, P. C. Arck and R. Paus, "Hair Growth Inhibition by Psychoemotional Stress: A Mouse Model for Neural Mechanisms in Hair Growth Control," Experimental Dermatology, Vol. 15, No. 15, 2006, pp. 1-13. doi:10.1111/j.0906-6705.2005.00372.x

[10] A. Slominski, J. Wortsman, A. Pisarchik, B. Zbytek, E. A. Linton, J. E. Mazurkiewicz and E. T. Wei, "Cutaneous Expression of Corticotropin-Releasing Hormone (CRH), Urocortin, and CRH Receptors," FASEB Journal, Vol. 15, No. 10, 2001, pp. 1678-1693. doi:10.1096/fj.00-0850rev

[11] X. Zhang, M. Yu, W. Yu, J. Weinberg, J. Shapiro and K. J. McElwee, "Development of Alopecia Areata is Associated with Higher Central and Peripheral Hypothalamicpituitary-Adrenal Tone in the Skin Graft Induced C3H/HeJ Mouse Model," Journal of Investigative Dermatology, Vol. 129, No. 6, 2009, pp. 1527-1538. doi:10.1038/jid.2008.371

[12] M. Baldari, M. Montinari, M. Guarrera and A. Rebora, "Trichodynia is a Distinguishing Symptom of Telogen Effluvium," Journal of the European Academy of Dermatology and Venereology, Vol. 23, No. 6, 2009, pp. 733734. doi:10.1111/j.1468-3083.2009.03201.x

[13] Y. Q. Cao, P. W. Mantyh, E. J. Carlson, A. M. Gillespie, C. J. Epstein and A. I. Basbaum, "Primary Afferent Tachykinins Are Required to Experience Moderate to Intense Pain," Nature, Vol. 392, No. 6674, 1998, pp. 390 394. doi:10.1038/32897

[14] R. Amann, R. Schuligoi, P. Holzer and J. Donnerer, "The Non-Peptide NK1 Receptor Antagonist SR140333 Produces Long-Lasting Inhibition of Neurogenic Inflammation, But Does Not Influence Acute Chemo- or Thermonociception in Rats," Naunyn-Schmiedeberg's Archives of Pharmacology, Vol. 352, No. 2, 1995, pp. 201-205. doi:10.1007/BF00176775

[15] Y. Akasaka, K. Abe, T. Sato and H. Inoue, "Regulation of Neurokinin-1 Receptor Messenger RNA Expression in Synovial Fibroblasts of Patients with Rheumatoid Arthritis," Neuropeptides, Vol. 39, No. 5, 2005, pp. 467-474. doi:10.1016/j.npep.2005.07.005

[16] S. Simeonidis, I. Castagliuolo, A. Pan, J. Liu, C. C. Wang, A. Mykoniatis, A. Pasha, L. Valenick, S. Sougioultzis, D. Zhao and C. Pothoulakis, "Regulation of the NK-1 Receptor Gene Expression in Human Macrophage Cells via an NF-Kappa B Site on Its Promoter," Proceedings of the National Academy of Sciences of the United States of America, Vol. 100, No. 5, 2003, pp. 2957-2962. doi: $10.1073 /$ pnas. 0530112100

[17] P. C. Arck, B. Handjiski, E. Hagen, R. Joachim, B. F. Klapp and R. Paus, "Indications for a 'Brain-Hair Follicle Axis (BHA)': Inhibition of Keratinocyte Proliferation and Up-Regulation of Keratinocyte Apoptosis in Telogen Hair Follicles by Stress and Substance P," The EMBO Journal, Vol. 5, No. 13, 2001, pp. 2536-2538. 
[18] R. Ruckert, G. Lindner, S. Bulfone-Paus and R. Paus, "High-Dose Proinflammatory Cytokines Induce Apoptosis of Hair Bulb Keratinocytes in Vivo," British Journal of Dermatology, Vol. 143, No. 5, 2000, pp. 1036-1039. doi:10.1046/j.1365-2133.2000.03784.x

[19] H. Gali-Muhtasib, A. Roessner and R. Schneider-Stock, "Thymoquinone: A Promising Anti-Cancer Drug from Natural Sources," International Journal of Biochemistry and Cell Biology, Vol. 38, No. 8, 2006, pp. 1249-1253. doi:10.1016/j.biocel.2005.10.009

[20] D. R. Worthen, O. A. Ghosheh and P. A. Crooks, "The in vitro Anti-Tumor Activity of Some Crude and Purified Compounds of Black Seed, Nigella sativa L," Anticancer Research, Vol. 18, No. 3A, 1998, pp. 1527-1532.

[21] F. M. Al-Awadi and K. A. Gumma, "Studies on the Activity of Individual Plants of an Antidiabetic Plant Mixture," Acta Diabetologica Latina, Vol. 24, No. 1, 1987, pp. 37-41. doi:10.1007/BF02732051

[22] B. H. Ali and G. Blunden, "Pharmacological and Toxicological Properties of Nigella sativa," Phytotherapy Research, Vol. 17, No. 1, 2003, pp. 299-305. doi:10.1002/ptr.1309

[23] A. Mohamed, D. M. Afridi, O. Garani and M. Tucci, "Thymoquinone Inhibits the Activation of NF-kB in the Brain and Spinal Cord of Experimentally Autoimmune Encephalomyelitis," Biomedical Sciences Instrumentation, Vol. 41, 2005, pp. 388-393.

[24] L. A. Garza, Y. Liu, Z. Yang, B. Alagesan, J. A. Lawson, S. M. Norberg, D. E. Loy, T. Zhao, H. B. Blatt, D. C. Stanton, L. Carrasco, G. Ahluwalia, S. M. Fischer, G. A. FitzGerald and G. Cotsarelis, "Prostaglandin D2 Inhibits Hair Growth and Is Elevated in Bald Scalp of Men with Androgenetic Alopecia," Science Translational Medicine, Vol. 4, No. 126, 2012, pp. 126-134.

[25] R. P. Hill, J. W. Haycock and C. A. Jahoda, "Human Hair Follicle Dermal Cells and Skin Fibroblasts Show Differential Activation of NF- $\kappa \mathrm{B}$ in Response to Pro-Inflammatory Challenge," Experimental Dermatology, Vol. 21, No. 2, 2012, pp. 158-160. doi:10.1111/j.1600-0625.2011.01401.x

[26] J. H. Han, O. S. Kwon, J. H. Chung, K. H. Cho, H. C. Eun and K. H. Kim, "Effect of Minoxidil on Profileration and Apoptosis in Dermal Papilla Cells of Human Hair Follicle," Journal of Dermatological Science, Vol. 34, No. 2, 2004, pp. 91-98. doi:10.1016/j.jdermsci.2004.01.002

[27] E. S. Friedman, P. M. Friedman, D. E. Cohen and K. Washenik, "Allergic Contact Dermatitis to Topical Minoxidil Solution: Etiology and Treatment," Journal of the American Academy of Dermatology, Vol. 46, No. 2, 2002, pp. 309-312. doi:10.1067/mjd.2002.119104

[28] D. E. Cohen, "New Vehicles for Delivery of Drugs to the Scalp," Dermatology Focus, Vol. 25, No. 2, 2006, pp.
2-3.

[29] A. G. Messenger and J. Rundegren, "Minoxidil: Mechanisms of Action on Hair Growth," British Journal of Dermatology, Vol. 150, No. 2, 2004, pp. 186-194. doi:10.1111/j.1365-2133.2004.05785.x

[30] E. S. El Daly, "Protective Effect of Cysteine and Vitamin E, Crocus sativus and Nigella sativa Extracts on Cisplatininduced Toxicity in Rats," Journal de Pharmacie de Belgique, Vol. 53, No. 2, 1998, pp. 87-95.

[31] T. Khanna, F. A. Zaidi and P. C. Dandiya, "CNS and Analgesic Studies on Nigella sativa," Fitoterapia, Vol. 5, No. 1, 1993, pp. 407-410.

[32] F. Vaillancourt, P. Silva, Q. Shi, H. Fahmi, J. C. Fernandes and M. Benderdour, "Elucidation of Molecular Mechanisms Underlying the Protective Effects of Thymoquinone against Rheumatoid Arthritis," Journal of Cellular Biochemistry, Vol. 112, No. 1, 2011, pp. 107-117. doi:10.1002/jcb.22884

[33] R. El Mezayen, M. El Gazzar, M. R. Nicolls, J. C. Marecki, S. C. Dreskin and H. Nomiyama, "Effect of Thymoquinone on Cyclooxygenase Expression and Prostaglandin Production in a Mouse Model of Allergic Airway Inflammation," Immunology Letters, Vol. 106, No. 1, 2006, pp. 72-81. doi:10.1016/j.imlet.2006.04.012

[34] N. Chehl, G. Chipitsyna, Q. Gong, C. J. Yeo and H. A. Arafat, "Antiinflammatory Effects of the Nigella sativa Seed Extract, Thymoquinone, in Pancreatic Cancer Cells," The Official Journal of the International Hepato Pancreato Biliary Association (Oxford), Vol. 11, No. 5, 2009, pp. 373-381.

[35] C. Deloche, O. de Lacharrière, C. Misciali, B. M. Piraccini, C. Vincenzi, P. Bastien, I. Tardy, B. A. Bernard and A. Tosti, "Histological Features of Peripilar Signs Associated with Androgenetic Alopecia," Archives of Dermatological Research, Vol. 295, No. 10, 2004, pp. 422-428. doi:10.1007/s00403-003-0447-y

[36] A. Steinmann, M. Schatzle, M. Agathos and R. Breit, "Allergic Contact Dermatitis from Black Cumin (Nigella sativa) oil after topical use," Contact Dermatitis, Vol. 36, No. 5, 1997, pp. 268-269. doi:10.1111/j.1600-0536.1997.tb00219.x

[37] S. Zedlitz, R. Kaufmann and W. H. Boehncke, "Allergic Contact Dermatitis from Black Cumin (Nigella sativa) Oil-Containing Ointment," Contact Dermatitis, Vol. 46, No. 3, 2002, pp. 188. doi:10.1034/j.1600-0536.2002.460318.x

[38] M. Yousefi, B. Barikbin, M. Kamalinejad, E. Abolhasani, A. Ebadi, S. Younespour, M. Manouchehrian and S. Hejazi, "Comparison of Therapeutic Effect of Topical Nigella with Betamethasone and Eucerin in Hand Eczema," Journal of the European Academy of Dermatology and Venereology, 2012, in Press. doi:10.1111/jdv.12033 